\title{
Two Cases of Lower Extremity Compartment Syndrome after Posterior Urethroplasty
}

Lollo L ${ }^{1 *}$, Ivashkov $\mathrm{Y}^{2}$, Grabinsky A ${ }^{1}$, Ramaiah $\mathrm{R}^{2}$

${ }^{1}$ Associate Professor, University of Washington, Seattle, WA, USA.

${ }^{2}$ Assistant Professor, University of Washington, Seattle, WA, USA.

\begin{abstract}
Acute lower extremity compartment syndrome following surgery in the lithotomy position is uncommon but is a surgical emergency causing irreversible damage if suspected and requires emergent decompressive fasciotomy. This report describes compartment syndrome following bulbo-urethroplasty. Patient A developed lower extremity pain with absent dorsiflexion 9 hours after surgery and Patient B experienced calf pain immediately after surgery. One year after fasciotomy A had permanent drop foot and B had peroneal nerve distribution numbness. Shared risk factors that placed the patients at risk were obesity and prolonged surgical times. Emerging concepts aimed at improving care for patients with compartment syndrome are discussed.
\end{abstract}

\begin{abstract}
*Corresponding Author:
Loreto Lollo MD, Seattle, WA 98195-6540, USA.

Tel: 2067442881

Fax: 2067446240

E-mail: lollomd@uw.edu
\end{abstract}

Associate Professor, Department of Anesthesiology and Pain Medicine, University of Washington, Box 3565401959 NE Pacific Street, BB-1469

Received: August 11, 2015

Accepted: September 19, 2015

Published: September 23, 2015

Citation: Lollo L, Ivashkov Y, Grabinsky A, Ramaiah R (2015) Two Cases of Lower Extremity Compartment Syndrome after Posterior Urethroplasty. Int J Anesth Res. 3(8), 158-161. doi: http://dx.doi. org $/ 10.19070 / 2332-2780-1500039$

Copyright: Lollo $\mathbf{L}^{\circ}$ 2015. This is an open-access article distributed under the terms of the Creative Commons Attribution License, which permits unrestricted use, distribution and reproduction in any medium, provided the original author and source are credited.

\section{Introduction}

Lower extremity compartment syndrome (CS) is characterized by severe pain resulting from increased interstitial pressure within the closed compartments of the calf musculature that impairs local circulation and left untreated results in irreversible peripheral nerve and muscle ischemia [1]. CS can occur in other anatomic regions including the arm, hand, foot, and gluteal area. Although an infrequent complication of severe tissue trauma including fracture and crush injury, it has been associated with minor injuries, and uncommon iatrogenic causes have been identified [2]. Acute CS is a surgical emergency treated by decompressive fasciotomy (DF) without delay to prevent permanent disability, amputation and death [3]. The diagnosis of acute CS is based on careful clinical examination. Intra-compartmental pressure (iCP) measurement, and monitoring if indicated, is a recommended clinical practice in equivocal situations [4]. Values of iCP greater than 30 $\mathrm{mm} \mathrm{Hg}$ above diastolic blood pressure are an absolute indication for emergent DF [4]. Lower leg CS following orthopedic and urologic operative procedures that place patients in the lithotomy position is an infrequent occurrence [5-7]. This report summarizes perioperative events and long term outcomes for acute CS following urethroplasty. A review of emerging diagnostic, interventional and prognostic strategies aimed at improving patient care following this complication is included.

Institutional Review Board approval was granted by the University of Washington Human Subjects Division.

\section{Case Reports}

The pertinent demographic and clinical data for both patients is summarized in Table 1. Timing of the surgical and anesthetic events for both patients is outlined in Figure1. Patient reported postoperative pain scores as a function of time are graphed in Figure 2.

Patient A reported a history of hypercholesterolemia, gastro-esophageal reflux disease (GERD), untreated obstructive sleep apnea (OSA), and occasional ethanol use. The patient's daily medications included hydro-chlorthiazide, losartan, metformin, glipizide, simvastatin and omeprazole. Ketorolac and oxycodone were prescribed for occasional severe migraine headaches and zolpidem for insomnia. Two prior uneventful urological procedures within the preceding year were done in the lithotomy position with anesthetic times for these surgical interventions 137 minutes and 95 minutes respectively.

Total intraoperative fluid administered for the urethroplasty procedure was $2350 \mathrm{ml}$ of crystalloid and estimated blood loss was $250 \mathrm{ml}$. Urinary output could not be monitored because the surgical procedure was performed on the urethra. Intraoperative hypotension during the course of the operation was treated with intravenous bolus doses of 100 micrograms (mcg) of phenylephrine with a total cumulative dose of $700 \mathrm{mcg}$ administered. 
Table 1. Patient Demographics.

\begin{tabular}{|c|c|c|c|c|c|c|}
\hline Patient & Age (years) & ASA & BMI & Nicotine Use & Diabetes & Hypertension \\
\hline A & 49 & 3 & 42.3 & Ex-smoker for $15 \mathrm{yrs}$ & Non-Insulin Dependent & Yes \\
\hline B & 47 & 1 & 35.3 & Ex-smoker for $18 \mathrm{yrs}$ & No & No \\
\hline
\end{tabular}

(ASA - American Society of Anesthesiology Physical Status Classification. BMI - Body Mass Index)

Figure 1. Surgical and Anesthesia Time Events (Anesthesia Start Time = 0 minutes. A - Patient A. B - Patient B.).

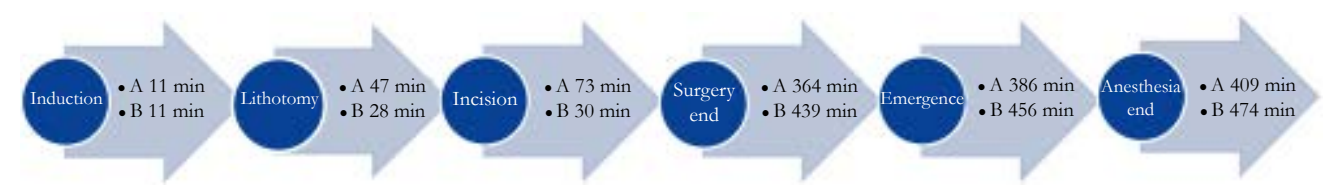

Figure 2. Plot of postoperative pain scores for patients and time after arrival in the recovery area.

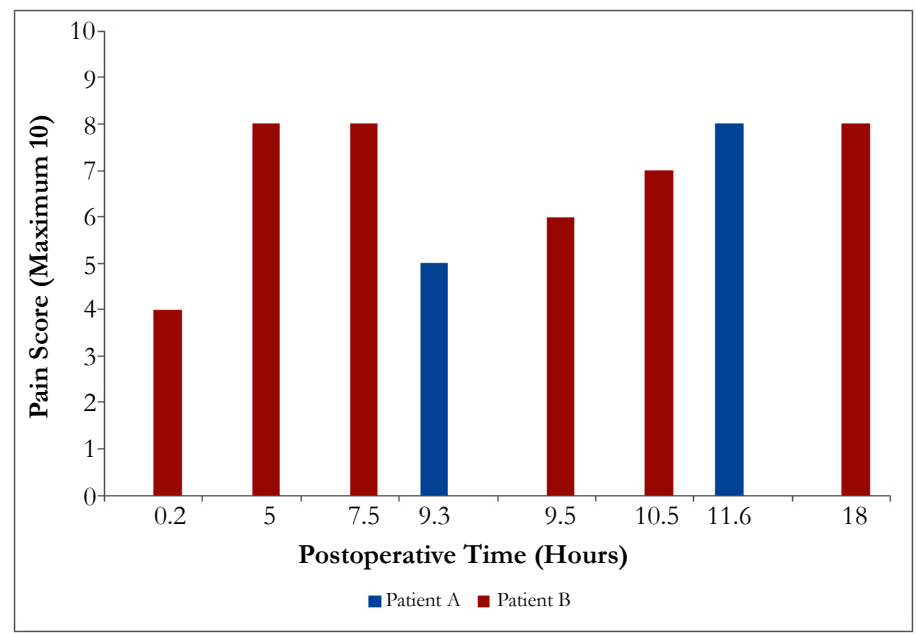

During the initial postoperative period the patient had an episode of restlessness and delirium that abated after incremental administration of $3 \mathrm{mg}$ of haloperidol and $100 \mathrm{mcg}$ fentanyl. The patient subsequently had difficulty oxygenating and was admitted to the intensive care unit (ICU) for monitoring of respiratory status. Pain was adequately controlled until at 9 hours and 20 minutes after arrival to the recovery room (PACU) when he reported bilateral leg cramps. During assessment at 9 hours and 45 minutes after surgery the patient reported pain in the anterior aspect of both legs and inability to move the left foot. Examination revealed a tense left lateral compartment and absence of left toe dorsiflexion. The pain persisted and escalated to a level that was intractable to analgesic medications. Orthopedic consultation was emergently obtained at which time CS was suspected and the patient underwent bilateral lower extremity 4 compartment decompressive fasciotomy 16 hours after arrival in the PACU.

Laboratory investigations demonstrated a venous lactate level of $3.7 \mathrm{mg} / \mathrm{dl}$ one hour after arrival in the PACU. Serial electrolyte measurements including potassium, blood urea nitrogen and creatinine were within normal limits throughout the hospital admission.

Serum creatine kinase $(\mathrm{CPK})$ and myogolobin $(\mathrm{Mb})$ levels were 43,000 units/ $\mathrm{ml}$ and 18,020 units/ $\mathrm{ml}$ when the patient reported escalating pain 12 hours after arrival in the PACU. Repeat enzyme levels were 37,400 units $/ \mathrm{ml}$ for CPK and 4420 units $/ \mathrm{ml}$ for $\mathrm{Mb}$ at 16 hours after DF. Due to concern for rhabdomyolysis and previous compromised respiratory status, the patient remained in the ICU for 48 hours after surgical decompression of the leg compartments and was then discharged to an acute surgical floor.

The patient required vacuum assisted wound therapy and another 4 surgeries for further debridement of the calf muscles until delayed wound closure was achieved 17 days after DF. The final disposition was home discharge at 19 days instead of the originally planned 23 hour admission.

At follow up clinic appointments the patient reported a baseline pain score of 4 at 30 days after discharge that declined to 3 at the 6 months postoperative visit, at which time he was not taking any analgesic medications.

Neurologic examination of the patient at the 6 month follow up visit was significant for a left peroneal nerve palsy with persistent foot drop treated with a fixed ankle foot orthotic (AFO) to prevent equinus contracture of the extremity. The patient reported being satisfied with the outcome and had resumed full time employment in sales and marketing.

Patient B consumed ethanol occasionally and 15 months previously had undergone an uneventful urologic procedure of 145 minutes duration in the lithotomy position.

Total intraoperative crystalloid fluid administered during the urethroplasty was $3500 \mathrm{ml}$ and estimated blood loss was $400 \mathrm{ml}$. Urinary output could not be assessed due to the nature of the procedure on the urethra. No episodes of hypotension occurred and 
no vasopressor medications were administered.

The patient reported right leg pain upon arrival in the PACU. Urgent orthopedic consultation was obtained and monitoring of compartments was commenced. The compartments were initially assessed as "soft" during an evaluation 5 hours after arrival in PACU. Re-assessment at 7.5 hours after PACU arrival the compartments were recorded as "firm" and the patient reported worsening pain on inversion of the right foot. Over the ensuing hours the patient reported the right leg pain was worsened by movement and noted globally decreased sensation below the knee. The patient reported continuous escalation of right leg pain that was not relieved by analgesic medication which had been partially successful in its treatment earlier. The iCPs were assessed using a Stryker compartment module 18 hours after arrival in the PACU. The anterior and lateral compartment iCPs were 34 and $36 \mathrm{~mm} \mathrm{Hg}$ with a delta $\mathrm{p}$ (difference from diastolic pressure) over $15 \mathrm{~mm} \mathrm{Hg}$ and neurologic examination demonstrated decreased sensation in the right sural, superficial peroneal and deep peroneal nerve distributions.

The patient underwent an emergent DF of the right leg anterior and lateral compartments 19.5 hours after initial arrival in the PACU. The fasciotomy wounds were treated with negative pressure wound vacuum therapy and delayed primary closure 3 days later. Discharge to home was on the fourth hospital day for this originally planned outpatient procedure. Serum laboratory investigations were normal throughout hospital admission. CPK and $\mathrm{Mb}$ levels were not assessed.

The patient reported no pain and no analgesic requirements at 30 days and one year after discharge. Neurologic findings demonstrated persistent numbness of the right deep and superficial peroneal nerves. The patient had returned to full time employment in education.

\section{Discussion}

Risk factors associated with acute postoperative CS following lithotomy positioning include large body habitus, excessive blood loss, peripheral vascular disease and operative times over 4 hours in duration $[8,9]$. Both patients in this case were obese and had surgery that lasted over 4 hours and had uneventful previous operations done in the lithotomy positon that were under 2.5 hours in duration. Patient A was diabetic and microvascular disease associated with this condition could have contributed to the worse long term outcome that he experienced. Pre-existing neuropathy or other neurologic disease can predispose patients to more severe nerve injury [10]. The remote history of cigarette smoking in both patients was an unlikely contributing factor to the subsequent development of CS.

Secondary renal injury resulting from rhabdomyolysis and myoglobinuria is a recognized complication of compartment syndrome resulting from prolonged surgery in the lithotomy position but this did not occur in either of the two patients reported [11].

Consensus for diagnosis, treatment and subsequent wound management of acute CS is lacking [12]. Continuous ICP monitoring is recommended but this measurement technique is prone to technical errors $[13,14]$. Non-invasive assessments being perfected that allow rapid and accurate evaluation of compartment tissue perfusion include near-infrared absorption, ultrasound measurement of tissue elastance, and muscle glucose concentration [15-
17]. In animal studies, negative pressure vacuum assisted wound closure techniques causes local tissue damage that results in delayed and altered healing [18].

The nerve damage resulting from ischemia or secondary fibrotic contractures and compression leads to weakness, paralysis, anesthesia, hypoesthesia and hyperesthesia distal to the point of injury [19]. Surgical decompression is associated with long-term problems of health-related quality of life particularly in relation to pain and discomfort [20]. Persistent pain in fasciotomy wounds at long term follow up occurs in $10 \%$ and one or more dystrophic signs associated with complex regional pain syndrome are present in 25 to $40 \%$ of patients [21]. Long term sequelae of acute CS may necessitate further surgical intervention including contracture release of the tendo Achilles, gastrocnemius recession, tenotomy for relief of claw toe deformity, nerve decompression or re-implantation, and in extreme cases amputation of the extremity [22]. Interventions aimed at more rapid and standardized diagnosis of acute CS and patient specific wound treatment strategies following fasciotomy will result in improved outcomes from this complication.

\section{References}

[1]. Via Ag, Oliva F, Spoliti M, Maffulli N (2015) Acute Compartment Syndrome. Muscles, Liagments, Tendons J 5(1): 18-22.

[2]. Frink M, Hildebrand F, Krettek C, Brand J, Hankemeier S (2010) Compartment syndrome of the lower leg and foot. Clin Orthop Relat Res 468(4): 940-950.

[3]. Raza H, Mahapatra A (2015) Acute compartment syndrome in orthopedics: causes, diagnosis and management. Adv Orthop 1-8.

[4]. Garner MR, Taylor SA, Gausden E, Lyden JP (2014) Compartment syndrome: diagnosis, management, and unique concerns in the twenty-first century. HSS J 10(2): 143-152.

[5]. Hsu KL, Chang CW, Lin CJ, Chang CH, Su WR, et al. (2015) The dangers of hemilithotomy positioning on traction tables: case report of a well-leg drop foot after contralateral femoral nailing. Patient Saf Surg 9(1): 1-4.

[6]. Simms MS, Terry TR (2005) Well leg compartment syndrome after pelvic and perineal surgery in the lithotomy position. Postgrad Med J 81(958): 534-536.

[7]. Bauer EC, Koch N, Janni W, Bender HG, Fleisch MC (2014) Compartment syndrome after gynecologic operations: evidence from case reports and reviews. Eur J Obstet Gynecol Reprod Biol 173: 7-12.

[8]. Bauer EC, Koch N, Erichsen CJ, Juettner T, Rein D, et al. (2014) Survey of compartment syndrome of the lower extremity after gynecologic surgery. Langenbecks Arch Surg 399(3): 343-348.

[9]. Chow CE, Friedell ML, Freeland MB, Dejesus S (2007) A pitfall of protracted surgery in the lithotomy Position: lower extremity compartment syndrome. Am Surg 73(1): 19-21.

[10]. Brull R, Hadzic A, Reina MA, Barrington MJ (2015) Pathophysiology and etiology of nerve injury following peripheral nerve blockade. Reg Anesth Pain Med 40(5): 479-490.

[11]. Vijay MK, Vijay P, Kundu AK (2011) Rhabdomyolysis and myogloginuric acute renal failure in the lithotomy/exaggerated lithotomy position of urogenital surgeries. Urol Ann 3(3): 147-50.

[12]. Donaldson J, Haddad B, Khan WS (2014) The pathophysiology, diagnosis and current management of acute compartment syndrome. Open Orthop J 8: 185-193.

[13]. McQueen MM, Duckworth AD, Aitken SA, Court-Brown CM (2013) The estimated sensitivity and specificity of compartment pressure monitoring for acute compartment syndrome. J Bone Joint Surg Am 95(8): 673-677.

[14]. Large TM, Agel J, Holtzman DJ, Benirschke SK, Krieg JC (2015) Interobserver variability in the measurement of lower leg compartment pressures. J Orthop Trauma 29(7): 316-321.

[15]. Cathcart CC, Shuler MS, Freedman BA, Reno LR, Budsberg SC (2014) Correlation of near-infrared spectroscopy and direct pressure monitoring in an acute porcine compartmental syndrome model. J Orthop Trauma 28(6): 365-369.

[16]. Sellei RM, Hingmann SJ, Kobbe P, Weber C, Grice JE (2015) Compartment elasticity measured by pressure-related ultrasound to determine patients "at risk" for compartment syndrome: an experimental in vitro study. Patient Saf Surg 9(1): 4. 
[17]. Doro CJ, Sitzman TJ, O’Toole RV (2014) Can intramuscular glucose levels diagnose compartment syndrome? J Trauma Acute Care Surg 76(2): 474478.

[18]. Wilkin G, Khogali S, Garbedian S, Slagel B, Blais S, et al. (2014) Negativepressure wound therapy after fasciotomy reduces muscle-fiber regeneration in a pig model. J Bone Joint Surg Am 96(16): 1378-1385.

[19]. Packer JD, Day MS, Nguyen JT, Hobart SJ, Hannafin JA, et al. (2013) Functional outcomes and patient satisfaction after fasciotomy for chronic exertional compartment syndrome. Am J Sports Med 41(2): 430-436.
[20]. Han F, Daruwalla ZJ, Shen L, Kumar VP (2015) A prospective study of surgical outcomes and quality of life in severe foot trauma and associated compartment syndrome after fasciotomy. J Foot Ankle Surg 54(3): 417-423.

[21]. Irion V, Magnussen RA, Miller TL, Kaeding CC (2014) Return to activity following fasciotomy for chronic exertional compartment syndrome. Eur J Orthop Surg Traumatol 24(7): 1223-1228.

[22]. Perkins ZB, Yet B, Glasgow S, Cole E, Marsh W, et al. (2015) Meta-analysis of prognostic factors for amputation following surgical repair of lower extremity vascular trauma. Br J Surg 102(5): 436-450. 